\title{
Neutrino physics with SHiP
}

\author{
Eric van Herwijnen* \\ CERN \\ E-mail: eric.van.herwijnendcern.ch
}

SHIP is a new general purpose fixed target facility, whose Technical Proposal has been recently reviewed by the CERN SPS Committee. It recommended that the experiment proceed further to a Comprehensive Design phase. In its initial phase, the $400 \mathrm{GeV}$ proton beam extracted from the SPS will be dumped on a heavy target with the aim of integrating $2 \times 10^{20}$ POT (Protons On Target) in 5 years. A dedicated detector, based on a long vacuum tank followed by a spectrometer and particle identification detectors, will allow probing a variety of models with light long-lived exotic particles and masses below $\mathscr{O}(10) \mathrm{GeV} / \mathrm{c}^{2}$. The main focus will be the physics of the so-called Hidden Portals. The sensitivity to Heavy Neutrinos will allow to probe for the first time the mass range between the kaon and the charm meson mass, and a range of couplings for which Baryogenesis and active neutrino masses could also be explained. Another dedicated detector will allow the study of neutrino cross-sections and angular distributions. $v_{\tau}$ deep inelastic scattering cross sections will be measured with a statistics 1000 times larger than currently available, allowing the extraction of the $F_{4}$ and $F_{5}$ structure functions, never measured so far.

38th International Conference on High Energy Physics

3-10 August 2016

Chicago, USA

${ }^{*}$ Speaker. 


\section{The objective of SHiP}

The discovery of the Higgs boson has been a triumph for the Standard Model of particle physics. In addition, despite subsequent intensive searches at the LHC and elsewhere, no significant deviations from the Standard Model have been found. However, there are a number of experimental facts that cannot be explained by the Standard Model, such as for example: neutrino masses and oscillations, dark matter, inflation and the baryonic asymmetry of the universe (BAU).

There are many theoretical models that explain these facts by introducing very weakly interacting new particles with masses up to a few $\mathrm{GeV} / \mathrm{c}^{2}$. But there is no guidance where to look for them. SHiP will try to find experimental support for some of these models.

\section{Extensions of the Standard Model}

It is possible that some of the particles responsible for the BSM problems have not been observed due to their extremely feeble interactions, rather than their heavy masses. Even in the scenarios in which BSM physics is related to high-mass scales, many models contain degrees of freedom with suppressed couplings that stay relevant at much lower energies.

\subsection{Portals}

It could be that some of the new particles do not interact directly with the SM sector. These "hidden sectors" may nevertheless be accessible via sufficiently light particles ("mediators"), which are coupled to SM particles via renormalizable interactions with small dimensionless coupling constants ("portals"). There are 3 types of portal, depending on the mass dimension of the SM singlet operator: vector (dark photons), scalar (hidden scalars) and neutrino (HNLs).

In these models, the production branching ratios of hidden sector particles are $\mathscr{O}\left(10^{-10}\right)$, with $\mathrm{c} \tau \sim \mathscr{O}(\mathrm{km})$ and they would travel unperturbed through matter.

\subsection{The $v$ Minimal Standard Model ( $v$ MSM)}

Interestingly, many current experiments and observations can be explained by a minimal extension of the Standard Model: the addition of three right-handed heavy neutral leptons (HNLs) (see Figure 2.2, [1]). A light $\mathscr{O}(1 \mathrm{keV}) N_{1}$ serves as dark matter candidate, mass-degenerate $\mathscr{O}\left(100 \mathrm{MeV} / \mathrm{c}^{2}-\right.$ few $\left.\mathrm{GeV} / \mathrm{c}^{2}\right) \mathrm{N}_{2,3}$ give mass to the active neutrinos via the see-saw mechanism, and $N_{2,3}$ account for leptogenesis and hence explain baryogenesis by increased $\mathrm{CP}$ violation.

In the $v$ MSM, HNL production takes place in semi-leptonic decays from charm $\left(M_{H N L}<\right.$ $\left.2 \mathrm{GeV} / \mathrm{c}^{2}\right)$ and beauty $\left(M_{H N L}<5 \mathrm{GeV} / \mathrm{c}^{2}\right)$, see Figure 2(a). Some examples of decays into SM particles are shown in Figure 2(c) and branching ratios in Figure 2(b)(see [2]).

\section{The SHiP detector}

\subsection{Experimental requirements}

Because the hidden particles are expected to be predominantly accessible through the decays of heavy hadrons, the facility is designed to maximise their production and detector acceptance, while providing the cleanest possible environment. With $2 \times 10^{20}$ protons on target, the charm production at the SPS exceeds any existing and planned facility (see Table 1). 


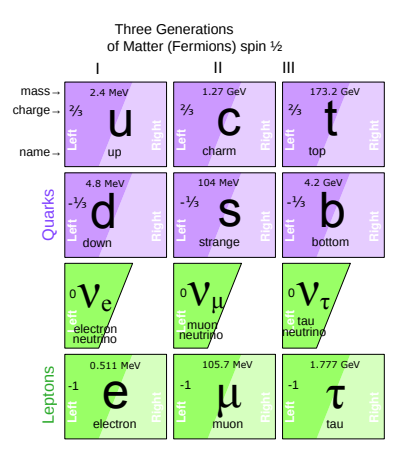

(a) The Standard Model

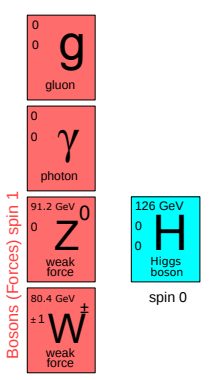

Figure 1: Adding three right handed Majorana HNLs to the Standard Model
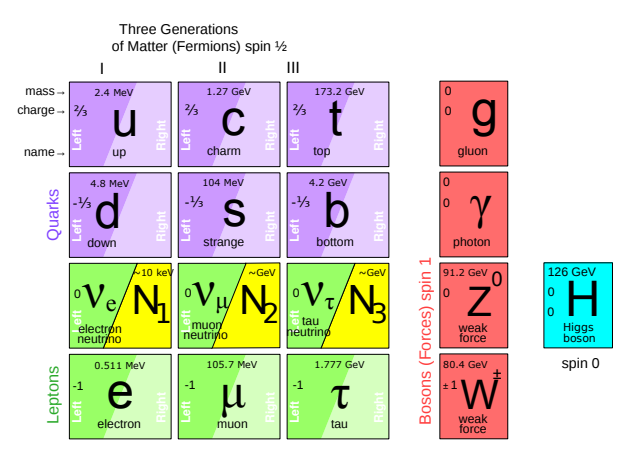
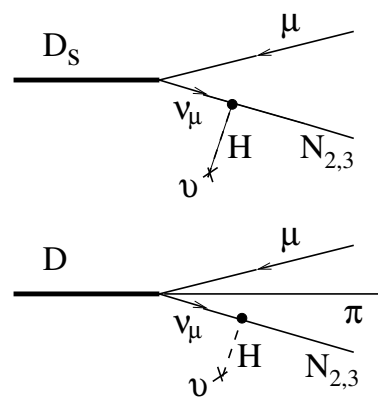

(a) HNL production

$$
\begin{aligned}
& \operatorname{Br}\left(D \rightarrow H N L X \sim 10^{-8}-10^{-12}\right) \\
& \operatorname{Br}\left(B \rightarrow H N L X \sim 10^{-12}-10^{-16}\right)
\end{aligned}
$$$$
\begin{aligned}
& \operatorname{Br}(H N L \rightarrow \mu(e) \pi) \sim 0.1-50 \% \\
& \operatorname{Br}(H N L \rightarrow \mu(e) \rho) \sim 0.5-20 \% \\
& \operatorname{Br}\left(H N L \rightarrow \mu(e) v_{e}\right) \sim 1-10 \%
\end{aligned}
$$

(b) HNL production/decay BRs
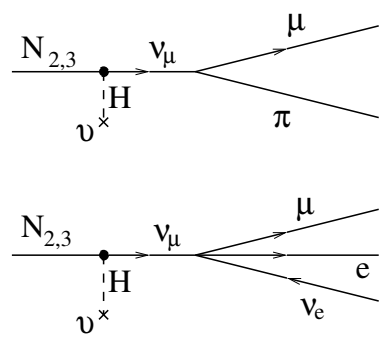

(c) HNL decays

Figure 2: HNL production from charm/beauty (a) and HNL decays (c)

Table 1: Production of charm at the LHC, SPS and Fermilab

\begin{tabular}{|l|l|l|}
\hline Facility & Energy & $c \bar{c}$ in $3-4$ years \\
\hline LHC-HL & $14 \mathrm{TeV}, \mathscr{L} \sim 1 a b^{-1}$ & $\sim 2 \times 10^{16}$ \\
SPS & $400 \mathrm{GeV}, 2 \times 10^{20}$ POT & $\gtrsim 10^{18}$ \\
Fermilab & $120 \mathrm{GeV}$ & $8 \times$ smaller $\sigma_{\mathrm{c} \bar{c}}$ \\
\hline
\end{tabular}

\subsection{Detector design}

Since the hidden sector particles originating from charm and beauty have a significant $p_{\mathrm{T}}$, the detector should be placed as close as possible to the target (see Figure 3).

A critical component of SHiP is the muon shield, which deflects the high flux of muon background away from the detector. The detector is designed to fully reconstruct the exclusive decays of hidden particles and to reject the background down to below 0.1 events in the sample of $2 \times 10^{20}$ protons on target.

The detector consists of a large magnetic spectrometer located downstream of a $50 \mathrm{~m}$-long and $5 \times 10 \mathrm{~m}$-wide decay volume. To suppress the background from neutrinos interacting in the fiducial volume, the decay volume is maintained under a vacuum. The spectrometer is designed to accurately reconstruct the decay vertex, mass and impact parameter of the decaying particle at the target. A set of calorimeters followed by muon chambers provide identification of electrons, 


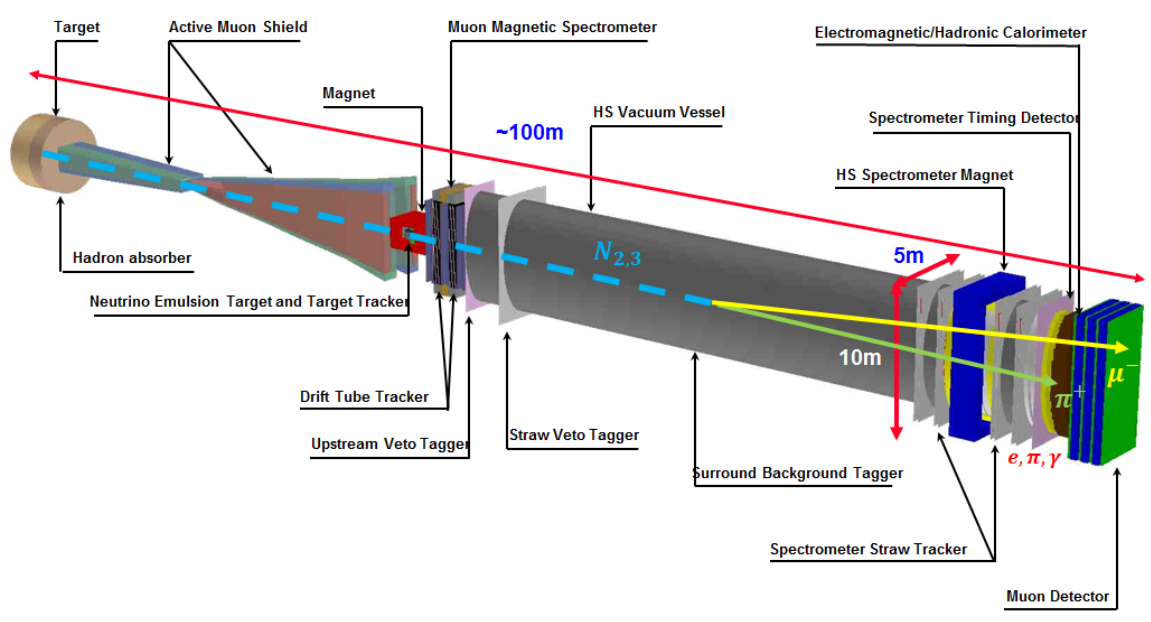

Figure 3: The SHiP detector

photons, muons and charged hadrons. A dedicated high-resolution timing detector measures the coincidence of the decay products, which allows the rejection of combinatorial backgrounds.

The decay volume is surrounded by background taggers to detect neutrino and muon inelastic scattering in the surrounding structures, which may produce long-lived SM $V^{0}$ particles, such as $\mathrm{K}_{\mathrm{L}}$, etc. The experimental facility is also ideally suited for studying interactions of tau neutrinos. It will therefore host an emulsion cloud chamber based on the Opera concept, upstream of the hidden-particle decay volume.

\section{Expected yields}

In the case of HNLs, the yield depends on the hierarchy of the active neutrino masses and on the relative strength of the HNL couplings to the three SM flavours $U_{e}^{2}, U_{\mu}^{2}, U_{\tau}^{2}$. For the normal hierarchy of active neutrino masses, theoretical work suggests $U_{e}^{2}: U_{\mu}^{2}: U_{\tau}^{2} \sim 1: 16: 3.8[2]$. This scenario, with a total coupling to the SM of $U^{2}=9.3 \cdot 10^{-9}$ and a HNL mass of $1 \mathrm{GeV} / \mathrm{c}^{2}$, was chosen as a benchmark scenario to investigate SHiP's acceptance in detail.

The expected yields for the channel $D \rightarrow \mu H N L X, H N L \rightarrow \mu \pi$ for $U_{\mu}^{2}=10^{-8}, 10^{-7}$ are 120,12000 respectively.

\subsection{Sensitivities}

The sensitivities of SHiP to HNL, dark photon and hidden scalars are shown in Figure 4.1. From these figures it can be seen that SHiP probes a unique range of couplings and masses.

\subsection{Neutrino physics}

With $5 v_{\tau}$ candidates observed by Opera [3] and 9 by Donut [4], the $v_{\tau}$ is the least known SM particle. The $\overline{v_{\tau}}$ has not yet been observed. 


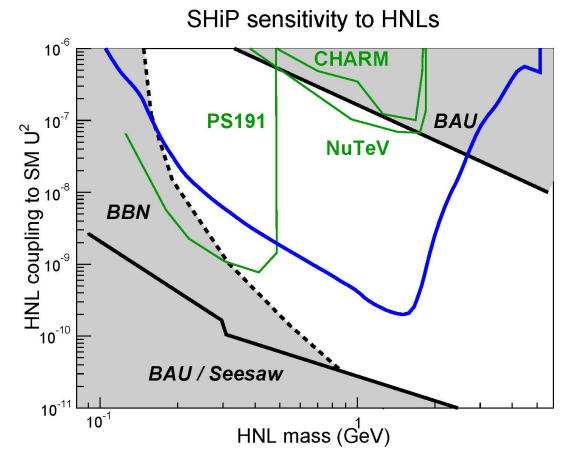

(a) HNL

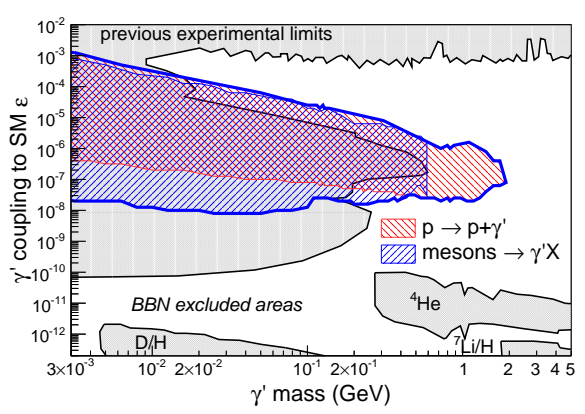

(b) Dark photon

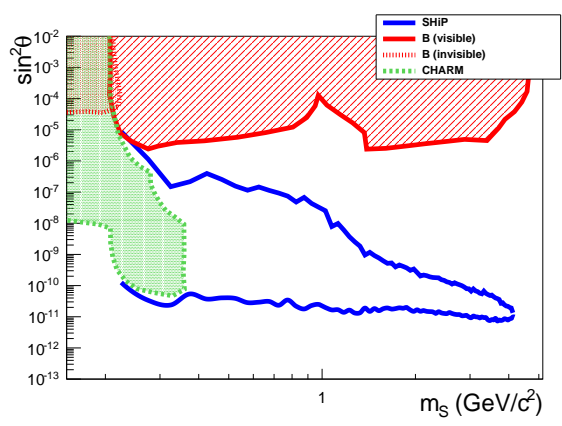

(c) Hidden scalars

Figure 4: SHiP sensitivities (blue) compared to theoretical and experimental limits

At SHiP we expect to produce (over 5 years of running) $5.7 \times 10^{15} v_{\tau}$ and $\bar{v}_{\tau}$ candidates via $p N \rightarrow X D_{s}\left(\rightarrow \tau \nu_{\tau}\right)$. To detect these particles, a "mini-Opera" emulsion spectrometer is placed between the $\mu$-shield and the decay vessel, see Figure 5. With these fluxes we expect $\sim 10^{4}\left(10^{3}\right) v_{\tau}\left(\overline{v_{\tau}}\right)$, $\sim 10^{5} v_{e}\left(\overline{v_{e}}\right)$ and $\sim 10^{6} v_{\mu}\left(\overline{v_{\mu}}\right)$ interactions. This will enable the first observation of $\overline{v_{\tau}}$, the first independent measurement of $\sigma_{v_{\tau}}$ and $\sigma_{\bar{v}_{\tau}}$ cross-sections and the first evaluation of the structure functions $\mathrm{F}_{4}$ and $\mathrm{F}_{5}$ which are not accessible with other neutrinos.

\section{Status of the project}

The SPSC has positively reviewed the Technical Proposal [5] and the CERN Research Board has recommended SHiP to proceed with a Comprehensive Design Study. A report will be given to the Physics Beyond Colliders working group so that it can provide input to the next European Strategy Group.

During the comprehensive design phase the layout of the experimental facility and the geometry of the detectors will be further optimized. This involves a detailed study of the muon-shield magnets and the decay volume. It also comprises revisiting the neutrino background in the fiducial volume, together with the background detectors, to decide on the required type of technology for evacuating the decay volume.

The SHiP collaboration currently consists of almost 250 members from 47 institutes in 15 countries. In only two years, the collaboration has formed and taken the experiment from a rough 


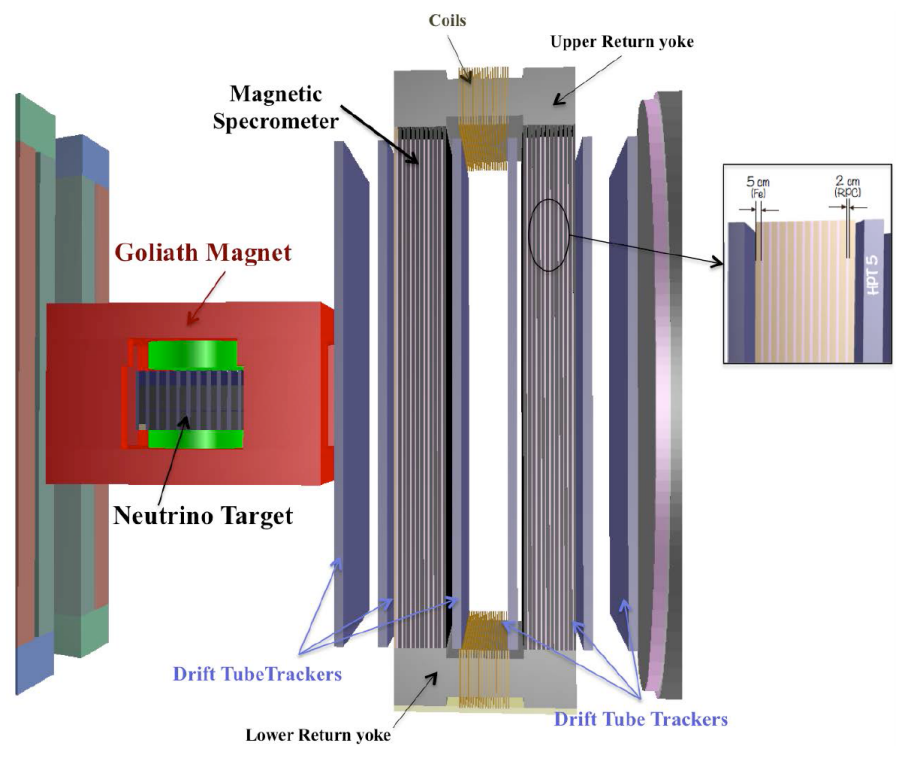

Figure 5: The tau neutrino detector

idea in the Expression of Interest to an already mature design in the TP. The SHiP physics case was demonstrated to be very strong by a collaboration of more than 80 theorists in the SHiP Physics Proposal [6].

\section{Conclusions}

The intensity frontier greatly complements the search for new physics at the LHC. Major improvements and new results are expected during the next decade in neutrino and flavour physics, proton-decay experiments and measurements of the electric dipole moments. CERN will be well-positioned to make a unique contribution to exploration of the hidden-particle sector with the SHiP experiment at the SPS.

\section{References}

[1] T. Asaka and M. Shaposhnikov, The vMSM, dark matter and baryon asymmetry of the universe, Phys.Lett.,B620, 17-26, (2005). doi: 10.106/j.physletb.2005.06.020.

[2] D. Gorbunov and M. Shaposhnikov, How to find neutral leptons of the vMSM?, 2007, arXiv:0705.1729

[3] R. Acquafredda et al., The OPERA experiment in the CERN to Gran Sasso neutrino beam, JINST, 4, P04018, (2009). doi: 10.1088/1748-0221/4/04/P04018.

[4] K. Kodama et al., Observation of tau neutrino interactions, Phys.Lett. B504, 218-224, (2001). doi:10.1016/S0370-2693(01)00307-0.

[5] The SHiP Collaboration, Technical Proposal. An Experiment to Search for Hidden Particles (SHiP) at the SPS, April 2015, CERN-SPSC-2015-016.

[6] S. Alekhin et al., A facility to Search for Hidden Particles at the CERN SPS: the SHiP physics case, Rep. Prog. Phys. 79, 124201, (2016). doi:10.1088/0034-4885/79/12/124201. 